\title{
Papers
}

\section{Effects of euthanasia on the bereaved family and friends: a cross sectional study}

Nikkie B Swarte, Marije L van der Lee, Johanna G van der Bom, Jan van den Bout, A Peter M Heintz

\begin{abstract}
Objective To assess how euthanasia in terminally ill cancer patients affects the grief response of bereaved family and friends.

Design Cross sectional study.

Setting Tertiary referral centre for oncology patients in Utrecht, the Netherlands.

Participants 189 bereaved family members and close friends of terminally ill cancer patients who died by euthanasia and 316 bereaved family members and close friends of comparable cancer patients who died a natural death between 1992 and 1999 .

Main outcome measures Symptoms of traumatic grief assessed by the inventory of traumatic grief, current feelings of grief assessed by the Texas revised inventory of grief, and post-traumatic stress reactions assessed by the impact of event scale.

Results The bereaved family and friends of cancer patients who died by euthanasia had less traumatic grief symptoms (adjusted difference - 5.29 (95\% confidence interval -8.44 to -2.15$)$ ), less current feeling of grief (adjusted difference 2.93 (0.85 to $5.01)$ ); and less post-traumatic stress reactions (adjusted difference $-2.79(-5.33$ to -0.25$)$ ) than the family and friends of patients who died of natural causes. These differences were independent of other risk factors.

Conclusions The bereaved family and friends of cancer patients who died by euthanasia coped better with respect to grief symptoms and post-traumatic stress reactions than the bereaved of comparable cancer patients who died a natural death. These results should not be interpreted as a plea for euthanasia, but as a plea for the same level of care and openness in all patients who are terminally ill.
\end{abstract}

\section{Introduction}

Grief is a normal reaction to the death of a loved one and normally does not require any professional help. Traumatic grief refers to situations where grief symptoms take too long or too short, are too intense or not intense enough, or come too late. ${ }^{1}$ Depending on the definition, $10-20 \%$ of bereaved people will suffer from traumatic grief.

Sudden loss, loss of a child or a partner, low self esteem, low internal control, lack of religion or spiritual belief, lack of social support, low education, and young age have been identified as risk factors for developing traumatic grief. ${ }^{2}$ Unnatural death, such as suicide, can cause severe grief reactions in family members. ${ }^{3}$ As euthanasia is also considered as an unnatural death, it has been suggested that euthanasia may induce traumatic grief. ${ }^{1}$ However, the grief experienced by family members in suicide cases differs from grief after euthanasia, mainly because the relatives of the latter have had the opportunity to say goodbye, which is seldom the case in suicides. ${ }^{4}$ Physician assisted suicide should be expected to resemble euthanasia on this point, because it will also usually be announced.

In the Netherlands euthanasia is carried out on about 3200 people a year, of whom $80 \%$ have cancer. ${ }^{56}$ So far, no studies have been made of how euthanasia in terminally ill cancer patients affects their bereaved family and friends. ${ }^{7}$ The aim of this study was to examine the effects of euthanasia in terminal cancer patients on grief among bereaved family and friends.

\section{Participants and methods}

\section{Study population}

We conducted a cross sectional study among the bereaved families and friends of cancer patients who had died, either by euthanasia or natural causes, in the University Medical Center Utrecht between 1992 and 1999. Euthanasia was defined as "the intentional termination of the life of a patient at his or her request by a physician.. ${ }^{8}$ This definition excludes termination of life without the request of the patient, as well as alleviation of pain and other symptoms with drugs that finally hasten the patient's death. All patients who died by euthanasia in our hospital during 1992-9 were centrally registered, and we selected them as patients for our study. For each patient who died by euthanasia, we selected two control patients from all cancer patients who died from natural causes in our hospital during the same period. To achieve comparability between these groups, we matched control patients by age at death, by year of death, and by sex. During 1992-9, 95 patients died by euthanasia in our hospital, of whom 89 were oncology patients. We were unable to contact the bereaved family and friends of 11 of these 89 patients because of incorrect mailing addresses, leaving 78 patients in the study. We therefore selected 156 control patients from the 1360 oncology patients who died naturally in our hospital during the same period.
Department of Gynaecology, F05.829, University Medical Center Utrecht, PO Box 85500, 3508 GA Utrecht,

Netherlands

Nikkie B Swarte research fellow A Peter M Heintz professor of gynaecological gynaecologic
oncology

Department of Clinical Psychology,

University of

Utrecht

Marije L van der

Lee

research fellow

Jan van den Bout professor of clinical psychology

Julius Centre for Health Sciences and Primary Care, University Medical Center Utrecht Johanna $\mathrm{G}$ van der Bom

assistant professor of clinical epidemiology Correspondence to: Nikkie B Swarte n.swarte@azu.nl

bmj.com 2003;327:189 
The study population consisted of bereaved people who were immediate family members (partner, parent, child, or sibling) or friends who were with the patient at the moment of death, were informed about the cause of death, were aged 18-85 years, and were fluent in Dutch. The physician who treated each dying patient approached the family members through the person who was registered as the contact name in the medical files. These contacts were asked to participate in this study and to supply the names and addresses of the other immediate family members and of relatives and friends who were with the patient at the moment of death so that these individuals could also be approached. We sent a questionnaire to all potential participants a week after sending them a letter giving information on our study. With the questionnaire, we also sent a refusal form for people to return to indicate that they did not want to participate. To those who did not respond, we sent a reminder a month after sending the questionnaire.

\section{Measurements}

Between January 2000 and August 2001 we sent questionnaires to all participants. The questionnaire contained, beside demographic items, seven standardised questionnaires assessing grief symptoms, posttraumatic stress reactions, general wellbeing, depressive symptoms, and personality as well as a general questionnaire we composed about several aspects of the period of the patient's death.

We assessed grief with a Dutch version of the inventory of traumatic grief (ITG), a 29 item, self completed questionnaire with a high score indicating a greater risk for traumatic grief, ${ }^{9}$ and with the second subscale of the Texas revised inventory of grief (TRIG), which consists of 13 items and measures current reactions to loss with high scores representing less current grief symptoms. ${ }^{10}$ We used this second subscale of the Texas revised inventory of grief because we were interested in the symptoms and psychological distress at the moment of the measurement, avoiding any recall bias.

We measured post-traumatic stress reactions with the impact of event scale (IES), which can be divided into the subscales intrusion and avoidance. ${ }^{11}$ A high score indicates more post-traumatic stress reactions.

We assessed general wellbeing with the symptom checklist (SCL-90), a 90 item, self completed symptom inventory designed to measure psychiatric symptoms. ${ }^{12}$ A high score indicates less general wellbeing.

We assessed depressive moods with a Dutch version of the depressive adjective checklist (DACL) ${ }^{13}$ This consists of 22 "positive" words and 12 "negative" words. The total score is the number of "negative" words that are ticked plus the number of unticked "positive" words, with a high score indicating more depressive symptoms.

We assessed participants' personality with the generalised self-efficacy scale (SES), which measures self confidence, ${ }^{14}$ and with the Eysenck personality questionnaire (EPQ-RRS) $)^{15}$, of which we used the part that measures neuroticism.

The general questionnaire we composed contained questions such as "Do you think you have really been able to say goodbye to the patient?" with answers on a five point scale ranging from "Not at all" to "Yes completely." Another question concerned support from others: "Do you think you have received enough support from others after the death?"

We also reviewed the medical files of the patients who died by euthanasia and of the control patients and recorded their demographic characteristics, medical history, and symptoms and treatment during their last admission. The duration of illness was defined as the period in months between date of cancer being diagnosed and date of death. We defined the date when cancer became untreatable as the time when no curative options were left for treating the disease.

\section{Power calculation and statistical analysis}

Our primary end point was the inventory of traumatic grief. We calculated that, with an estimated mean score on the inventory of traumatic grief of 82 for the relatives of patients who died a natural death, we would need 300 relatives of patients who died naturally and 150 relatives of patients who died by euthanasia, to find, with a power of 0.90 and a two sided significance of 0.05 , a $10 \%$ increased or decreased score for the relatives of patients who died by euthanasia. We therefore tried to contact the relatives of all 78 patients who died by euthanasia between 1992 and 1999 and to contact the relatives of twice as many patients who died a natural death.

We calculated $\mathrm{P}$ values with either Student's $t$ test or, for discrete characteristics, with the Pearson $\chi^{2}$ test. We performed multilevel analysis with the program MLwiN 1.1. ${ }^{16}$ This statistical technique allowed for the dependency of measurements in hierarchically structured data (there is a positive correlation between responses from family members and close friends of the same dead person), whereas traditional multiple regression analysis presupposes the independency of observations. Grief symptoms, post-traumatic stress reactions, general wellbeing, and depressive symptoms were considered as continuous outcomes. When a questionnaire had more than $10 \%$ of the values missing, we did not include it in the analyses. For the other missing values, we used the mean values of the study population.

\section{Results}

Our results are based on returned questionnaires of the bereaved relatives and friends of 58 of the 78 patients who died by euthanasia and 114 of the 156 control patients who died naturally. Table 1 shows the characteristics and medical history of the dead patients. Because of the matching procedure, there was no apparent difference between patients who died by euthanasia and controls in age at death, year of death (described by the period between sending out the questionnaire and date of death), and sex. The duration of illness was longer for the patients who died by euthanasia, but the difference was not statistically significant. There was no difference in whether the cancer was untreatable at first diagnosis.

Of the 264 questionnaires we sent to the bereaved relatives and friends of cancer patients who died by euthanasia, 197 were completed and 189 (72\%) had less than $10 \%$ missing values. Of the 480 questionnaires sent to bereaved family and friends of the control patients, 392 were completed and $316(66 \%)$ had less than $10 \%$ missing values. The family members 
who did not complete the (whole) questionnaire or did not respond were more likely to be male $(51 \%)$ than those who responded ( $43 \%$ male) and they did not differ in their kind of kinship with the dead patient.

Table 2 presents the demographic characteristics of the bereaved relatives and friends. The relatives and friends of the euthanasia patients were less religious than the family and friends of the control patients, and their level of education was higher. Their relationship with the patients also differed: the family and friends of euthanasia patients consisted more of the subgroup defined as others (such as cousins, in-laws, or friends), whereas the family and friends of control patients consisted of more children and siblings.

Table 3 shows the differences in grief symptoms, post-traumatic stress reactions, general wellbeing, and depressive symptoms between the bereaved relatives and friends of euthanasia patients and those of control patients. For all questionnaires except the Texas revised inventory of grief a high score means more symptoms or complaints. We found a small but non-significant difference between the groups for the symptom checklist (relatives and friends of euthanasia patients scoring somewhat better on general wellbeing), and both groups scored similarly on the depressive adjective checklist. However, the relatives and friends of euthanasia patients had significantly lower scores on the inventory of traumatic grief and impact of event scale score and higher scores on the Texas revised inventory of grief. The percentage of bereaved people who fulfilled the criteria of traumatic grief was twice as high in the control group as in the relatives and friends of euthanasia patients $(5.7 \% v 2.1 \%)$, indicating that there was a clinically significant difference as well as a statistically significant one.

Educational level, the score on the Eysenck personality questionnaire neuroticism scale, and kinship were the most important confounders of the association between cause of death and grief symptoms and posttraumatic stress reactions. After adjustment for these factors, euthanasia was still associated with less severe symptoms and reactions. Adjustment for all other potential determinants for traumatic grief did not change these findings (table 3). However, adjustment for "saying goodbye" to patients considerably weakened the association between cause of death and grief symptoms or post-traumatic stress reactions (table 3).

\section{Discussion}

In this cross sectional study, the bereaved families and friends of cancer patients who died by euthanasia had less grief symptoms and post-traumatic stress reactions than the families and friends of comparable cancer patients who died in a natural way. Adjustment for all other determinants for traumatic grief, including the duration of illness, did not influence our findings. The bereaved family and friends of euthanasia death scored somewhat better on general wellbeing, while depressive symptoms were similar for both groups. The opportunity to say goodbye to the patient seemed to be an important determinant of less grief symptoms and partly explained the association between cause of death and grief or post-traumatic stress reactions.

The consequences of euthanasia on grief among the bereaved family and friends have been described
Table 1 Characteristics and medical history of 58 cancer patients who died from euthanasia in the University Medical Center Utrecht between 1992 and 1999 and 114 control cancer patients who died from natural causes. Values are numbers (percentages) unless specified otherwise

\begin{tabular}{lcc}
\multirow{2}{*}{ Characteristics } & \multicolumn{2}{c}{ Cause of death } \\
\cline { 2 - 3 } Female & Euthanasia (n=58) & Natural death $(\mathbf{n}=\mathbf{1 1 4})$ \\
\hline Mean $(95 \% \mathrm{Cl})$ age at death (years) & $37 / 58(64)$ & $70 / 114(61)$ \\
\hline Geometric mean $(95 \% \mathrm{Cl})$ length of illness (months) & $61(58$ to 64$)$ & $62(60$ to 64$)$ \\
\hline $\begin{array}{l}\text { Cancer untreatable at first diagnosis } \\
\text { Geometric mean }(95 \% \mathrm{Cl}) \text { period between date } \\
\text { questionnaire sent out and date of death (months) }\end{array}$ & $35(29$ to 22$)$ & $10(7$ to 13$)$ \\
\hline
\end{tabular}

by Van den Boom, who interviewed 60 relatives of 52 AIDS patients who had died. ${ }^{17}$ Twelve of these patients had died by euthanasia. He found no significant association between the prevalence of depression in the bereaved family and friends and the way of death. However, he did find that a complicated euthanasia process was associated with complicated grief and added distress to the bereaved family and friends. In our study complications in the euthanasia process did not occur; situations like that are more likely to occur when euthanasia is performed at home. ${ }^{18}$ To our knowledge, this is the first study on the association of euthanasia in patients with terminal cancer and the grief symptoms in family members.?

\section{Limitations of study}

Non-response may have influenced our findings. However, the responses from the relatives of the patients who died by euthanasia were somewhat higher than that from the relatives of patients who died naturally $(75 \% v 69 \%)$. People with greater feelings of grief are more prone to non-response than persons with less feelings of grief. ${ }^{19}$ Therefore, the differences we found are probably an underestimation rather than an overestimation from non-response. It is also possible that the contact people for the dead patients selected only those relatives and friends for the study who were coping well, so that those with more severe grief symptoms may not have been invited to participate. However, it is unlikely that this would be different for

Table 2 Demographic characteristics of the relatives and friends of 58 cancer patients who died by euthanasia in the University Medical Center Utrecht between 1992 and 1999 and of 114 control cancer patients who died from natural causes. Values are numbers (percentages) unless specified otherwise

\begin{tabular}{|c|c|c|c|}
\hline \multirow[b]{2}{*}{ Characteristics } & \multicolumn{2}{|c|}{ Cause of death } & \multirow[b]{2}{*}{$\begin{array}{l}\text { P value of } \\
\text { difference }\end{array}$} \\
\hline & $\begin{array}{l}\text { Euthanasia } \\
\qquad(\mathrm{n}=189)\end{array}$ & $\begin{array}{l}\text { Natural death } \\
(\mathrm{n}=316)\end{array}$ & \\
\hline Female & $102 / 189(54)$ & $184 / 316(58)$ & 0.4 \\
\hline Mean (SD) age (years) & $48(14)$ & $49(14)$ & 0.4 \\
\hline Religious & 89/184 (48) & 200/313 (64) & 0.01 \\
\hline \multicolumn{4}{|l|}{ Relationship with dead patient: } \\
\hline Spouse & 33/189 (17) & $61 / 316(19)$ & \multirow{5}{*}{$<0.01$} \\
\hline Parent & $4 / 189(2)$ & $4 / 316(1)$ & \\
\hline Offspring & $56 / 189(30)$ & $117 / 316(37)$ & \\
\hline Sibling & $27 / 189(14)$ & $66 / 316(21)$ & \\
\hline Other & $69 / 189(37)$ & $68 / 316(22)$ & \\
\hline \multicolumn{4}{|l|}{ Highest level of education: } \\
\hline Primary education & $10 / 187(5)$ & $22 / 311(7)$ & \multirow{6}{*}{$<0.01$} \\
\hline Lower vocational education & 14/187 (8) & 48/311 (15) & \\
\hline Lower or intermediate general education & 26/187 (14) & $58 / 311(19)$ & \\
\hline Higher general education & 28/187 (15) & $32 / 311(10)$ & \\
\hline Intermediate vocational education & $38 / 187(20)$ & $66 / 311(21)$ & \\
\hline Higher vocational education or university & $71 / 187(38)$ & $85 / 311(27)$ & \\
\hline
\end{tabular}


Table 3 Differences between the relatives and friends of 58 cancer patients who died by euthanasia in the University Medical Center Utrecht between 1992 and 1999 and of 114 control cancer patients who died from natural causes in scores for grief, post-traumatic stress reactions, general wellbeing, and depressive symptoms. Values are means $(95 \%$ confidence intervals) unless stated otherwise

\begin{tabular}{|c|c|c|c|c|c|c|}
\hline \multirow[b]{2}{*}{ Questionnaire } & \multicolumn{2}{|c|}{ Mean $(95 \% \mathrm{CI})$ scores by cause of death } & \multicolumn{4}{|c|}{ Difference $(95 \% \mathrm{CI})^{*}$} \\
\hline & Euthanasia $(n=189)$ & $\begin{array}{l}\text { Natural death } \\
\quad(n=316)\end{array}$ & Crude & $\begin{array}{c}\text { Adjusted for } \\
\text { education, EPQ, and } \\
\text { kinship with patient }\end{array}$ & Adjusted for all $\dagger$ & $\begin{array}{c}\text { Adjusted for } \\
\text { opportunity to "say } \\
\text { goodbye" }\end{array}$ \\
\hline Inventory of traumatic grief & 39 (38 to 41)‡ & $45(43$ to 47$) \ddagger$ & $-7.05(-10.64$ to -3.45$)$ & $-5.54(-8.95$ to -2.12$)$ & $-5.29(-8.44$ to -2.15$)$ & $-5.51(-9.29$ to -1.73$)$ \\
\hline Texas revised inventory of grief & 46 (44 to 48 ) & 42 (41 to 43 ) & 3.57 (1.23 to 5.91$)$ & 2.60 (0.27 to 4.92$)$ & 2.93 (0.85 to 5.01$)$ & 2.77 (0.31 to 5.24$)$ \\
\hline Impact of event scale & $13(11$ to 15$)$ & $18(16$ to 19$)$ & $-4.78(-7.66$ to -1.89$)$ & $-3.74(-6.54$ to -0.94$)$ & $-2.79(-5.33$ to -0.25$)$ & $-3.14(-6.09$ to -0.19$)$ \\
\hline Symptom checklist & 116 (111 to 120$)$ & 121 (118 to 125$)$ & $-5.62(-11.83$ to 0.59$)$ & $-2.43(-6.93$ to 2.07$)$ & $-1.61(-6.29$ to 3.08$)$ & $-2.46(-9.0$ to 4.08$)$ \\
\hline Depressive adjective checklist & 9 (8 to 10$)$ & $9(9$ to 10$)$ & $-0.04(-0.92$ to 0.84$)$ & $0.34(-0.42$ to 1.10$)$ & $0.40(-0.39$ to 1.19$)$ & $0.40(-0.53$ to 1.32$)$ \\
\hline
\end{tabular}

$E P Q=$ Eysenck personality questionnaire.

*Differences obtained by multilevel regression analysis, $95 \%$ confidence intervals calculated with robust standard errors.

†Adjusted for: education, EPQ score, kinship with dead patient, age, sex, self efficacy score, religion, support of bereaved, depression of dead patient, patient's age at death, patient's length of treatable illness, patient's duration of illness, period between questionnaire and patient's date of death, adequate pain treatment for patient, adequate treatment of gastrointestinal complaints for patient, other complaints of patient, and whether the cancer was treatable at time of diagnosis.

$\ddagger$ Geometric mean.

relatives of patients who died from natural causes and thus cannot explain our findings.

In the Netherlands many patients die at home (40\%), especially oncology patients (48\% of all cancer deaths). ${ }^{20}$ Our study population may therefore not be representative of relatives of all dead oncology patients. It is possible that people experienced death and bereavement in hospital in a different way than the relatives of cancer patients who died at home. We therefore have to restrict the interpretation of our findings to patients dying in hospital.

Extraneous factors that were not within the scope of this study might explain the differences we found between the two groups. For example, terminally ill patients who request euthanasia may have a different personality from those who do not, and this difference may also hold for their family members. We tried to take this into account by controlling for neuroticism, but other personality factors may have influenced the results.

Another difference between the two groups of patients surely must have been the capacity to acknowledge their prognosis. Since prognostic denial is most likely to arise in patients with underlying distress, ${ }^{21}$ and distress will have a negative effect on the wellbeing of the bereaved family and friends, this might also explain the differences between the two groups. ${ }^{22}$ Moreover, Chochinov also found an association between intense family contact and prognostic awareness. A possible explanation for our results is that family members may have colluded with the prognostic denial of deeply distressed patients in the group that died of natural causes. This may have had a detrimental effect on the grief process of these family members afterward..$^{23}$ There is a need for open awareness of impending death and for careful and thoughtful planning for where and how the death ought to occur. ${ }^{24}$

\section{Conclusion}

Possible explanations for less grief symptoms among the bereaved family and friends of cancer patients who died by euthanasia are: (a) the opportunity to say goodbye while these patients were generally still fully aware, an interpretation that our results support; $(b)$ the bereaved family and friends of these were probably more prepared for the way and day of the imminent death; and $(c)$ when a terminally ill patient requests euthanasia, family members and the patient are often able to talk openly about death. Our results should not

\section{What is already known on this topic?}

In the Netherlands 3200 patients die by euthanasia each year, and $80 \%$ have terminal cancer

The grieving process by friends and relatives can be long and painful, but the effect of euthanasia on this process has not been studied

\section{What this study adds}

Grief after the death of a terminally ill cancer patient by euthanasia was not more complicated for the family members than grief after a natural death

Having the opportunity to say goodbye to the patient was an important determinant of grief and post-traumatic stress reactions

be interpreted as a plea for euthanasia, but as a plea for the same level of care and openness in all patients who are terminally ill.

We thank all the family members who took part in the study. We also thank Cora Maas for her advice on multilevel analysis.

Contributors: NBS participated in designing the protocol, collected data, and participated in data analysis and interpretation. MLvdL participated in the data analysis and interpretation and in revising the manuscript. JGvdB participated in designing the protocol, data interpretation, and writing and revising the manuscript. JvdB initiated and designed the proto$\mathrm{col}$, and participated in writing and revising the paper. APMH participated in the design of the protocol and revising the manuscript. APMH is guarantor for the paper.

Funding: The study was funded by the Department of Gynaecology, University Medical Center Utrecht, and by the Research Institute for Psychology and Health.

Competing interests: None declared.

Ethical approval: The study was approved by the Medical Ethics Committee of the University Medical Center Utrecht.

1 Jacobs S, Mazure C, Prigerson H. Diagnostic criteria for traumatic grief. Death Stud 2000;24:185-99.

2 Stroebe W, Schut H. Risk factors in bereavement outcome: a methodological and empirical review. In: Stroebe MS, Hansson RO, Stroebe W, Schut H, eds. Handbook of bereavement research. Washington DC: American Psychological Association, 2001:613-45.

3 Kelly BJ, Varghese FT. Assisted suicide and euthanasia: what about the clinical issues? Aust N Z J Psychiatry 1996;30:3-8.

4 Swarte NB, Heintz AP. Euthanasia and physician-assisted suicide. Ann Med 1999;31:364-71.

5 Pijnenborg L, Van Delden JJ, Kardaun JW, Glerum JJ, Van der Maas PJ Nationwide study of decisions concerning the end of life in general practice in the Netherlands. BMJ 1994;309:1209-12. 
6 Van der Maas PJ, Van der Wal G, Haverkate I, de Graaff CL, Kester JG, Onwuteaka-Philipsen BD, et al. Euthanasia, physician-assisted suicide, and other medical practices involving the end of life in the Netherlands, 1990-1995. N Engl J Med 1996;335:1699-705.

Emanuel EJ. Euthanasia and physician-assisted suicide. Arch Intern Med 2002;162:142-52

Swarte NB, Heintz APM. Guidelines for an acceptable euthanasia procedure. Baillieres Clin Obstet Gynaecol 2000;15:313-21.

9 Boelen PA, Van den Bout J, de Keijser J, Hoijtink H. Reliability and validity of the Dutch version of the inventory of traumatic grief (ITG). Death Stud 2003;27:1-21.

10 Faschingbauer RRZS, DeVaul R. The Texas revised inventory of grief. In: Zisook S, ed. Biopsychosocial aspects of bereavement. Washington DC: Ameriress, 1987:127-38.

11 Horowitz MJ, Wilner N, Alvarez W. Impact of event scale: a measure of subjective stress. Psychosom Med 1979;41:209-18.

12 Arrindell WA, Ettema J. SCL-90. Manual for a multidimensional indicator of psychopathology. Lisse: Swets and Zeitlinger, 1986.

13 Lubin B. Adjective checklist for measurement of depression. Arch Gen Psychiatry 1965;12:57-62.

14 Wegner M, Schwarzer R, Jerusalem M. Generalized self-efficacy. In: Schwarzer R, ed. Measurement of perceived self-efficacy. Psychometric Scales for cross-cultural research. Berlin: Freie Universität Berlin, 1993.

15 Sanderman R, Arrindell WA, Ranchor AV, Eysenck HJ. Eysenck personality questionnaire. Groningen, University of Groningen, 1995.
16 Goldstein H, Rasbash J, Plewis I, Draper D, Browne W, Yang M, et al. A user's guide to MlwiN. London: University of London, 1998.

17 Van den Boom F. AIDS, euthanasia and grief. AIDS Care 1995;7(suppl 2):S175-85

18 Groenewoud JH, Van der Heide A, Onwuteaka-Philipsen BD, Willems DL, Van der Maas PJ, Van der Wal G. Clinical problems with the performance of euthanasia and physician-assisted suicide in the Netherlands. N Engl J Med 2000;342:551-6.

19 Stroebe MS, Stroebe W. Who participates in bereavement research? A review and empirical study. Omega 1989;20:1-29.

20 Van der Wal G, Dillmann RJ. Euthanasia in the Netherlands. BMJ 1994;308:1346-9.

21 Chochinov HM, Tataryn DJ, Wilson KG, Ennis M, Lander S. Prognostic awareness and the terminally ill. Psychosomatics 2000;41:500-4.

22 Valdimarsdottir U, Helgason AR, Furst CJ, Adolfsson J, Steineck G. The unrecognised cost of cancer patients' unrelieved symptoms: a nationwide follow-up of their surviving partners. BrJ Cancer 2002; 86:1540-5.

23 BrintzenhofeSzoc KM, Smith ED, Zabora JR. Screening to predict complicated grief in spouses of cancer patients. Cancer Pract 1999;7:233-9.

24 Koop PM, Strang V. Predictors of bereavement outcomes in families of patients with cancer: a literature review. Can J Nurs Res 1997;29:33-50.

(Accepted 17 June 2003) 